\title{
Crossover phenomena involving the dense $O(n)$ phase
}

\author{
Wenan Guo ${ }^{1, *}$ and Henk W. J. Blöte ${ }^{2,3}$ \\ ${ }^{1}$ Physics Department, Beijing Normal University, Beijing 100875, P.R. China \\ ${ }^{2}$ Instituut Lorentz, Leiden University, P.O. Box 9506, NL-2300 RA Leiden, The Netherlands \\ ${ }^{3}$ Faculty of Applied Sciences, Delft University of Technology, P.O. Box 5046, NL-2600 GA Delft, The Netherlands
}

(Received 1 November 2010; published 28 February 2011)

\begin{abstract}
We explore the properties of the low-temperature phase of the $\mathrm{O}(n)$ loop model in two dimensions by means of transfer-matrix calculations and finite-size scaling. We determine the stability of this phase with respect to several kinds of perturbations, including cubic anisotropy, attraction between loop segments, double bonds, and crossing bonds. In line with Coulomb gas predictions, cubic anisotropy and crossing bonds are found to be relevant and introduce crossover to different types of behavior. Whereas perturbations in the form of loop-loop attractions and double bonds are irrelevant, sufficiently strong perturbations of these types induce a phase transition of the Ising type, at least in the cases investigated. This Ising transition leaves the underlying universal low-temperature $\mathrm{O}(n)$ behavior unaffected.
\end{abstract}

DOI: 10.1103/PhysRevE.83.021115

PACS number(s): 05.50.+q, 64.60.Cn, 02.50.-r, 75.10.Hk

\section{INTRODUCTION}

The $\mathrm{O}(n)$ spin model is defined in terms of $n$-component spins on a lattice, with spin-spin interactions that satisfy $\mathrm{O}(n)$ symmetry that is, the model is isotropic in the $n$-dimensional spin-vector space. The cases $n=1,2$, and 3 correspond to the Ising, $X Y$, and Heisenberg models, respectively, but the significance of the $\mathrm{O}(n)$ model goes beyond that of these spin models. A loop expansion of the partition function of certain two-dimensional $\mathrm{O}(n)$ spin models $[1,2]$ leads to a system of nonintersecting loops, while the spin degrees of freedom are integrated out. The resulting loop gas is called the $\mathrm{O}(n)$ loop model, sometimes abbreviated to the $\mathrm{O}(n)$ model. It has only discrete degrees of freedom, but the spin dimensionality $n$ appears in the partition sum of the $\mathrm{O}(n)$ loop model as a continuously variable parameter. In the limit $n \rightarrow 0$, the model serves to describe the behavior of polymer configurations [3-5].

For some two-dimensional $\mathrm{O}(n)$ spin models, a mapping on a loop model is possible such that it yields the partition function in a form that enables the derivation of exact results [6-17]. These results show that there exist several "branches" of universality classes that continuously depend on the parameter $n$ for $-2 \leqslant n \leqslant 2$.

One of these branches describes the phase transition between the high-temperature disordered spin phase and the low-temperature phase, where the spins display longrange correlations. In the terms of the loop model, the high-temperature phase is characterized by small loops and a low loop density, and the low-temperature phase by a high loop density and the existence of a loop of divergent size.

The low-temperature phase appears to be more interesting than what one might expect on the basis of the known properties of the long-range-ordered $\mathrm{O}(1)$ or Ising model. For general $n$ in the interval $-2 \leqslant n \leqslant 2$, the low-temperature phase is still critical in the sense that the correlation

\footnotetext{
*waguo@bnu.edu.cn
}

functions display power-law behavior. Its universal properties are described by another exactly solved branch. Recently, an exact transformation was applied to map the low-temperature branch of the $\mathrm{O}(n)$ loop model onto a tricritical loop model that includes vacant sites [18]. This mapping was applied for the case of the honeycomb as well as for that of the square lattice. Since this tricritical $\mathrm{O}(n)$ model should have two more relevant temperature-like fields than the low-temperature branch, one may wonder whether these relevant directions have some physical meaning in the low-temperature $\mathrm{O}(n)$ phase.

The present work focuses on the stability properties of the low-temperature phase of the loop model with respect to several perturbations that move the loop model away from the exactly solvable point. These perturbations are as follows.

(1) An attractive potential associated with loop segments that collide at a vertex of the lattice.

(2) The introduction of double bonds, which allow some lattice edges to be covered by up to two loop segments.

(3) A cubic perturbation of the $\mathrm{O}(n)$ spin symmetry, which translates into the connection of four incoming loop segments at a vertex.

(4) Crossing bonds coupling $\mathrm{O}(n)$ spins, which correspond with crossing loop segments in the loop model, without affecting the $\mathrm{O}(n)$ symmetry of the corresponding spin model.

The existing results in the literature, in particular, from Coulomb gas theory $[19,20]$, predict, or at least suggest, the effects of these perturbations. Cubic deviations from $\mathrm{O}(n)$ symmetry were concluded to be irrelevant on the critical branch for $n<2$, and to be relevant on the $n<2$ lowtemperature branch [20]. Crossing bonds are predicted to be described by the same exponent, so that they should also be relevant in the low-temperature phase. Attractions between loop segments were, however, concluded [21] to be irrelevant in this phase.

Our present work purports to test the theoretical predictions numerically, by means of transfer-matrix calculations. In Sec. II we define the models under investigation, and summarize the relevant existing results. Section III explains 
the numerical procedures and Sec. IV presents the numerical results, concerning the phase diagram and the relevance or irrelevance of the various perturbations. We conclude with a discussion of the results in Sec. V.

\section{MODELS}

The $\mathrm{O}(n)$ spin model with pair interactions is described by the reduced Hamiltonian

$$
\mathcal{H}=-\frac{1}{k_{\mathrm{B}} T} \sum_{\langle i j\rangle} J\left(\vec{s}_{i} \cdot \vec{s}_{j}\right),
$$

where the sum is over all nearest-neighbor pairs, and the $\vec{s}_{i}$ are $n$-dimensional vectors whose label indicates the site number $i$. They are normalized as $\vec{s}_{i} \cdot \vec{s}_{i}=n$, and their integration measure is $\int d \vec{s}_{i}=1$. The function $J$ describes the pair energy as a function of the spin product and is usually chosen as a multiplicative constant, although other choices still preserve the $\mathrm{O}(n)$ symmetry. For the special choice $J(y)=k_{\mathrm{B}} T \ln (1+z y)$ the Hamiltonian becomes

$$
\mathcal{H}=-\sum_{\langle i j\rangle} \ln \left(1+z \vec{s}_{i} \cdot \vec{s}_{j}\right),
$$

where the parameter $z$ represents the coupling strength between neighboring $\mathrm{O}(n)$ spins, and can thus be understood as a measure of the inverse temperature. We consider the ferromagnetic case $z>0$. The partition integral of this model is

$$
Z=\left[\prod_{k} \int d \vec{s}_{k}\right] \prod_{\langle i j\rangle}\left(1+z \vec{s}_{i} \cdot \vec{s}_{i}\right),
$$

where the products are on the sites and on the nearest-neighbor pairs of the lattice, respectively.

\section{A. Loop model on the honeycomb lattice}

For the model on the honeycomb lattice, a graph expansion [2] of Eq. (3) expresses the partition function in terms of a sum over all configurations $\mathcal{G}$ of nonintersecting loops on the edges of the honeycomb lattice:

$$
Z_{\text {loop }}=\sum_{\mathcal{G}} z^{N_{b}} n^{N_{l}},
$$

where the graph $\mathcal{G}$ covers $N_{b}$ bonds of the lattice and consists of $N_{l}$ closed, nonintersecting loops. Each lattice edge may be covered by at most one loop segment. Exact analysis [6-9] appears possible for special values $z=z_{\mathrm{c}}$ given by

$$
z_{\mathrm{c}}=1 / \sqrt{2 \pm \sqrt{2-n}}, \quad-2 \leqslant n \leqslant 2,
$$

where the plus sign corresponds with a critical ordering transition separating the high-temperature phase from the low-temperature phase. The minus sign corresponds to the low-temperature $\mathrm{O}(n)$ phase. The solutions with a plus sign are called branch 1; those with a minus sign, branch 2. The exact results include the leading scaling dimensions.

These theoretical analyses are possible because of the special form of the spin-spin interaction $J$ in Eq. (1) and because each spin occurs, at most, to the third power in the expansion of Eq. (3).

For $n>2$ the model described by Eq. (4) no longer displays critical points resembling branch 1 or 2 , but there exists a line of critical points [22] resembling the hard-hexagon transition [23].

\section{B. Loop model on the square lattice}

Analogous to the case of the honeycomb lattice, an $\mathrm{O}(n)$ spin model can be defined such that it can be transformed into a system of nonintersecting loops [11,21] on the square lattice. In this case, the spins are located on the middle of the edges connecting the vertices of the $\mathrm{O}(n)$ loop model. The partition function of the latter model is a function of the loop weight $n$ and the vertex weights $u, v$, and $w$. The vertex weights of the square lattice $\mathrm{O}(n)$ model are defined in Fig. 1 .

The partition sum of the resulting loop model is simply written in terms of these weights as

$$
Z_{\text {loop }}=\sum_{\mathcal{G}} u^{N_{u}} v^{N_{v}} w^{N_{w}} n^{N_{l}} .
$$

The sum is over all graphs $\mathcal{G}$ consisting of nonintersecting loops on the square lattice, and $N_{u}, N_{v}$, and $N_{w}$ are the numbers of vertices with weights $u, v$, and $w$ respectively.

The resulting square-lattice $\mathrm{O}(n)$ loop model is solvable for special choices of the vertex weights [11]. The solution includes four branches of critical points, where "critical" refers to algebraic decay of correlations. These four branches form a one-parameter family, parametrized by an angle $\theta$ that is a four-valued function of the loop weight $n$. For branch $k$ (with $1 \leqslant k \leqslant 4$ ) the relation is

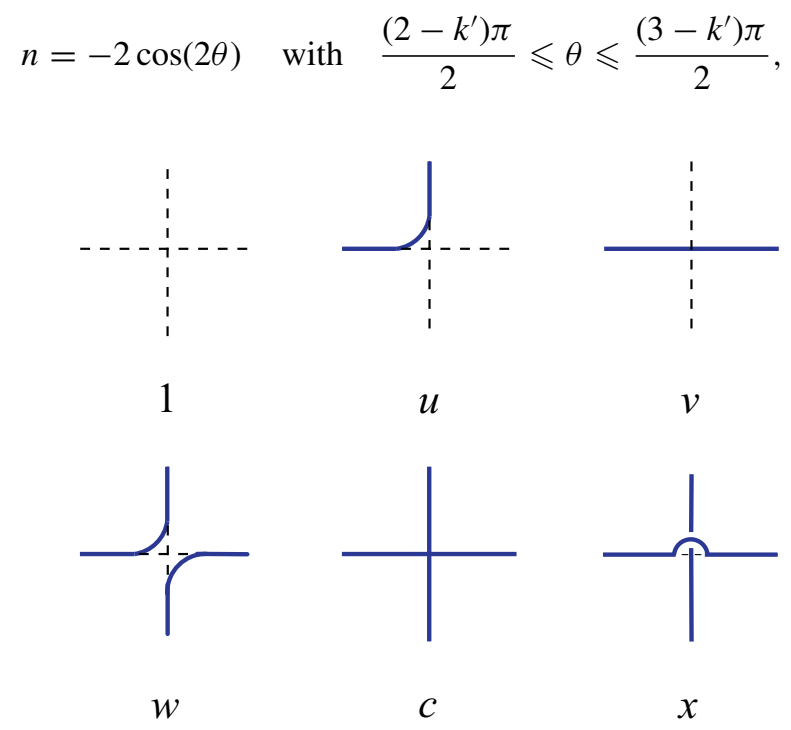

FIG. 1. (Color online) Vertex weights $u, v$, and $w$ of the $\mathrm{O}(n)$ loop model on the square lattice. They are normalized such that the empty vertex has weight 1 . In the models described by branches $1-4$, the same weights apply to rotated versions of the vertices shown. Also shown are additional vertices associated with perturbations of the model of Eq. (6), namely, cubic vertices with weight $c$ and crossingbond vertices with weight $x$. 
with

$$
k^{\prime}=2,1,3,4 \text { for } k=1,2,3,4 \text {, respectively. }
$$

The vertex weights are

$$
\begin{gathered}
u= \pm \frac{4 \sin (\theta / 2) \cos (\pi / 4-\theta / 4)}{2-\{1-2 \sin (\theta / 2)\}\{1+2 \sin (\theta / 2)\}^{2}}, \\
v= \pm \frac{1+2 \sin (\theta / 2)}{2-\{1-2 \sin (\theta / 2)\}\{1+2 \sin (\theta / 2)\}^{2}}, \\
w=\frac{1}{2-\{1-2 \sin (\theta / 2)\}\{1+2 \sin (\theta / 2)\}^{2}} .
\end{gathered}
$$

It appeared that, after the relabeling of $k^{\prime}$ by $k$, branches 1 and 2 share the universal properties of branches 1 and 2, respectively, on the honeycomb lattice. Branch 3 represents a multicritical point where the $\mathrm{O}(n)$ critical transition, a first-order transition, and an Ising transition merge. The Ising degrees of freedom can be understood in terms of dual spins on the faces of the square lattice, such that neighboring dual spins have the same sign only if they are separated by a loop segment [21]. The universal properties of branch 4 indicate a superposition of an Ising-like critical state and the low-temperature $\mathrm{O}(n)$ phase [21]. Thus branch 4 is interpreted as a point where the aforementioned Ising degrees of freedom undergo an ordering transition. A sketch of the resulting phase diagram, as conjectured in Ref. [21] and confirmed in Ref. [24] for the case $n=0$, is reproduced in Fig. 2.

The introduction of a sufficiently strong attractive potential between loop segments associated with the weight $w$ can, in principle, lead to an $\mathrm{O}(n)$ tricritical point [25]. The latter result applies only to the case $n=0$. This tricritical point is, however, of a different universal type than the multicritical point in the phase diagram in Fig. 2. Branches of tricritical points, parametrized by $n$, have been found for square $[18,26]$ and honeycomb [27] lattices with vacancies.

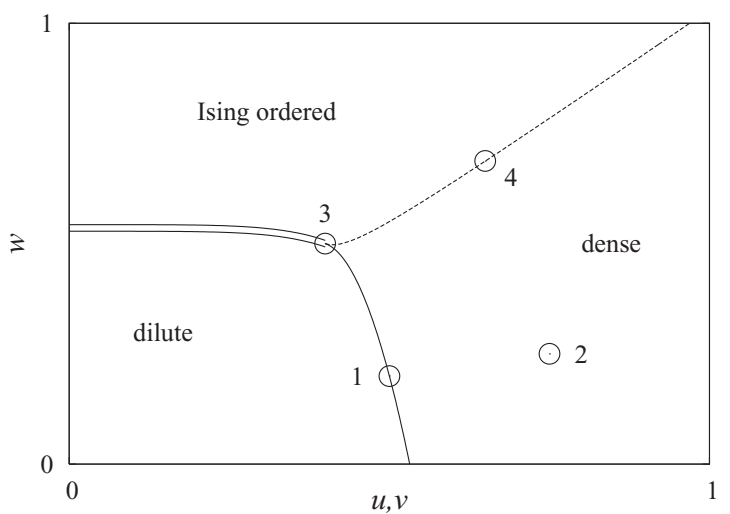

FIG. 2. Qualitative phase diagram of the $\mathrm{O}(n)$ loop model on the square lattice in a plane parametrized by the weights $u \approx v$ and $w$. Locations of the exactly solved points are indicated by an open circle and the corresponding branch number. The dilute $\mathrm{O}(n)$ loop phase corresponds to the disordered phase of the $\mathrm{O}(n)$ spin model, and the dense phase, to the low-temperature phase of the spin model. The solid curve represents the critical line of the $\mathrm{O}(n)$ ordering transition. The dense phase and the even denser Ising-ordered phase are separated by a line of Ising-like critical points (dashed line). The first-order transition between the disordered phase and the Ising-ordered lowtemperature phase is shown by the double line.

\section{The $n$-component cubic model}

In the face-cubic spin models, the spin vector is restricted to lie along one of $n$ Cartesian axes. Since it can still point in both directions of each axis, it has $2 n$ possible states. The spins lie on a lattice and have nearest-neighbor couplings of the form

$$
\mathcal{H}=-\sum_{\langle i j\rangle}\left[K \overrightarrow{s_{i}} \cdot \overrightarrow{s_{j}}+M\left(\overrightarrow{s_{i}} \cdot \overrightarrow{s_{j}}\right)^{2}\right] .
$$

A graph expansion of the $n$-component cubic model was described in Refs. [28] and [29]. The resulting partition sum thus depends, just as in the case of the $\mathrm{O}(n)$ model, continuously on $n$. The form of the interaction between the cubic spins is, as in Ref. [29], chosen such that $e^{M} \cosh K=1$, in which case the graph expansion contains only even vertices, that is, vertices that connect to an even number of neighbor sites. Thus, the graph expansion of the cubic model introduces a new vertex with four connected legs in comparison with the nonintersecting $\mathrm{O}(n)$ loop model. The graph representation of the partition function of this cubic model is

$$
Z_{\mathrm{cub}}=(2 n)^{N} \sum_{\mathcal{G}_{c}} z_{\mathrm{cub}}^{N_{b}} n^{N_{l}},
$$

where $z_{\text {cub }} \equiv n^{-1} e^{M} \sinh K, N$ is the number of sites of the lattice, and the sum is over all graphs $\mathcal{G}_{c}$ that contain only even vertices, that is, vertices connecting to zero, two, or four loop segments. The partition sum can be written in a form similar to Eq. (6), with $u=v=z_{\text {cub }}, w=0$, and an additional cubic four-leg vertex with weight $c=z_{\text {cub }}^{2}$.

Also, the Coulomb gas analysis of the $\mathrm{O}(n)$ model [20] uses four-leg vertices to describe a cubic perturbation. It predicts that cubic perturbations are irrelevant on the $\mathrm{O}(n)$ critical line for $n<2$. Numerical analyses of the scaling dimensions of the $n$-component cubic critical line $[28,29]$ do indeed confirm $\mathrm{O}(n)$ universal behavior. In contrast, the cubic perturbation is predicted to be relevant in the low-temperature phase. It may thus seem rather curious that the cubic model of Eq. (11), when defined on the honeycomb lattice, reduces exactly to the form of Eq. (4), so that the cubic perturbation plays no role. The low-temperature phase of the $n$-component cubic model of Eq. (11) on the honeycomb lattice is still in the universality class of the branch $2 \mathrm{O}(n)$ model for $n<2$. The exact results for the honeycomb $\mathrm{O}(n)$ model, including the critical point, apply as well to the $n$-component honeycomb cubic model of Eq. (11). For the square-lattice cubic model the critical point is not exactly known.

\section{The $O(n)$ model with crossing bonds}

The perturbation of the low-temperature $\mathrm{O}(n)$ phase by the introduction of a square-lattice vertex with crossing bonds is predicted to be relevant [6,20] and is thus expected to introduce crossover to different universal behavior. According to Jacobsen et al. [30], the generic $\mathrm{O}(n)$ low-temperature phase is described by the crossing-bond model in Refs. [31] and [32]. This equivalence indicates the existence of a magnetic dimension $X_{h}=0$, but attempts to verify this by finite-size scaling [30,31] suffer from poor convergence, which may be attributed to logarithmic factors. 


\section{E. Exact exponents}

The exact results for the critical exponents of the critical and the dense phase of the $\mathrm{O}(n)$ model can be conveniently expressed in terms of the Coulomb gas scaling dimensions $X\left(e_{1}, e_{2}, m_{1}, m_{2}\right)$ associated with two pairs $\left(e_{1}, m_{1}\right)$ and $\left(e_{2}, m_{2}\right)$ of electric and magnetic charges. The scaling dimension associated with these two pairs is [20]

$$
X\left(e_{1}, e_{2}, m_{1}, m_{2}\right)=-\frac{e_{1} e_{2}}{2 g}-\frac{m_{1} m_{2} g}{2},
$$

where $g$ is the coupling constant of the Coulomb gas. For branches 1 and 2 of the $\mathrm{O}(n)$ model it is related to the loop weight $n$ as

$$
g=1 \pm \frac{1}{\pi} \arccos \frac{n}{2},
$$

where the plus sign applies to branch 1 and the minus sign to branch 2 .

For the magnetic dimension $X_{h}$, one has $m_{1}=-m_{2}=1 / 2$ and $e_{1}=e_{2}=1-g$, so that

$$
X_{h}=1-\frac{1}{2 g}-\frac{3 g}{8} .
$$

In contrast with the $w$-type vertex, which is believed to be irrelevant in the dense phase of the $\mathrm{O}(n)$ loop model [21], the cubic and the crossing-bond vertices change the topology of the graph representation. In the language of the mapping on the Coulomb gas, they are described by the four-leg watermelon diagram, which translates into magnetic charges $m_{1}=-m_{2}=$ 2 [5]. The scaling dimensions $X_{c}$ of a cubic perturbation of the $\mathrm{O}(n)$ symmetry, and $X_{x}$ of crossing bonds, are thus

$$
X_{c}=X_{x}=1-\frac{1}{2 g}+\frac{3 g}{2} .
$$

For $n=2$ these perturbations are marginal, and for $n<2$ they are relevant on branch 2 .

The temperature dimension is [20]

$$
X_{t}=\frac{4}{g}-2,
$$

which is irrelevant for $n<2$ on branch 2 . It is expected to describe the effects of a variation of the vertex weights $u, v$, and $w$ with respect to the branch 2 point.

\section{TRANSFER-MATRIX METHOD}

The transfer-matrix technique is used to calculate the free energy density and the magnetic correlation length of $\mathrm{O}(n)$ models wrapped on a cylinder of a finite circumference of $L$ lattice units and of an infinite length. The free energy density of the system is

$$
f(L)=\frac{\zeta}{L} \ln \Lambda_{0}(L)
$$

where $\Lambda_{0}(L)$ is the leading eigenvalue of the transfer matrix $\mathbf{T}$, and $\zeta$ is the geometric factor, defined as the ratio of the unit of the finite size $L$ over the layer thickness corresponding to the action of $\mathbf{T}$. Thus, for the square lattice $\zeta=1$.

It is useful to divide the transfer matrix into two diagonal blocks or "sectors" as follows. When one cuts the cylinder through $L$ edges that are parallel to the axis of the cylinder, the number of dangling loop segments may be even or odd. It is obvious that the properties of evenness and oddness are conserved along the cylinder, so that the transfer matrix decomposes into an odd and an even sector. The eigenvalue $\Lambda_{0}(L)$ is the largest one in the even sector. The largest eigenvalue in the odd sector is denoted $\Lambda_{1}(L)$. The states of the odd sector describe the effect of an additional single loop segment running in the length direction of the cylinder. The mapping between the $\mathrm{O}(n)$ spin model and the loop model provides the interpretation that the odd sector describes the spin-spin correlation function along the cylinder. The magnetic correlation length $\xi_{h}(L)$ is thus inversely proportional to the logarithm of the gap in the eigenvalue spectrum of $\mathbf{T}$ :

$$
\xi_{h}^{-1}(L)=\zeta \ln \left[\Lambda_{0}(L) / \Lambda_{1}(L)\right] .
$$

The calculation of the eigenvalues $\Lambda_{0}(L)$ and $\Lambda_{1}(L)$ of $\mathbf{T}$ is still made subject to the condition that the associated eigenvectors possess translational symmetry; that is, the eigenvectors are invariant under the rotation of the cylinder over an angle $2 \pi / L$ about its axis. The translational symmetry is in line with the form of the partition sums given above and the periodic boundary conditions of a model on a cylinder. The correlation length $\xi_{h}(L)$ can be calculated numerically by the transfer-matrix method as a function of a parameter $P$ representing the distance (in some direction that remains to be specified) to a critical point or fixed point. Including this parameter in our notation, we define the scaled magnetic gap as

$$
X_{h}(P, L)=\frac{L}{2 \pi \xi_{h}(P, L)} .
$$

For models attracted by a conformally invariant fixed point, the scaled gap converges to the magnetic scaling dimension [33]. At a distance $P$ of the fixed point, finite-size scaling [34] then predicts

$$
X_{h}(P, L)=X_{h}+a P L^{2-X_{P}}+\cdots,
$$

where $X_{p}$ is the smallest scaling dimension of the scaling fields to which $P$ contributes, $a$ is an unknown amplitude, and the dots stand for corrections to the leading scaling behavior that vanish for $L \rightarrow \infty$. Since differentiation of Eq. (20) yields

$$
\frac{d X_{h}(P, L)}{d P}=a L^{2-X_{P}}+\cdots,
$$

it is possible to estimate $X_{P}$ if numerical data for $d X_{h}(P, L) / d P$ are available for a range of finite sizes $L$. These data can be obtained by numerical differentiation, that is, calculation of the scaled gap for several values of $P$, and subsequent fitting of a polynomial in $P$ through the scaled gaps.

The transfer-matrix construction for the $\mathrm{O}(n)$ model on the square lattice is described in Ref. [21], including the coding that defines the transfer-matrix index in terms of the "connectivities" describing the topology of the loop configuration at a cross section of the cylinder. A sparse-matrix decomposition allows the evaluation of the leading eigenvalues of transfer matrices with linear sizes up to a few times $10^{7}$ with the use of modest computer resources. Calculations for the $\mathrm{O}(n)$ model on a honeycomb lattice require a different sparse-matrix decomposition, which is explained in Ref. [35]. 
Furthermore,we also introduce two new types of vertices on the square lattice that generate a larger set of connectivities than those of the nonintersecting loop model. These are the cubic vertex and the crossing-bond vertex, included in Fig. 1. The introduction of the cubic vertex into the $\mathrm{O}(n)$ loop model leads to connections between the loops and thus leads to a larger set of connectivities in comparison with the loop model. The number of mutually connected dangling edges is no longer restricted to two but may also assume multiples of 2. The coding and decoding needed for the construction of the transfer matrix corresponding to the cubic model of Eq. (11) are described in Ref. [28].

Also, the presence of crossing bonds leads to an increase in the number of connectivities. While the dangling loop segments can only be connected pairwise, the "wellnestedness" property of the nonintersecting loop model is lost. This property implies that, if dangling bonds $i$ and $j$ are connected, and dangling bonds $k$ and $l$ are also connected, the situation $i<k<j<l$ is excluded. Once crossing-bond vertices are allowed, the loops get entangled, and the situation $i<k<j<l$ becomes possible. The coding of this larger set of connectivities by means of integers $1,2, \ldots$ is actually simpler than that of the well-nested connectivities [21]. The coding is determined by a set of rules specifying an ordering of these connectivities. For completeness, we describe an ordering including the non-well-nested $L$-point connectivities. It is useful to represent a connectivity $\alpha$ by an array of integers $\vec{i}_{\alpha} \equiv\left[i_{\alpha}(1), i_{\alpha}(2), \ldots, i_{\alpha}(L)\right]$, such that $i_{\alpha}(k)=i_{\alpha}(l)$ if and only if positions $k$ and $l$ are connected, that is, if dangling edges $k$ and $l$ are covered by dangling segments of the same loop. The special value $i_{m}=0$ represents a dangling edge not covered by a loop segment. The ordering of the connectivities, denoted by Greek symbols, is formulated in terms of these arrays of integers. The rules are as follows.

(1) For the $L_{\alpha}$-point connectivity $\alpha$, remove the integers with $i_{m}=0$ from the array $\vec{i}_{\alpha}$. This leads to an $L_{\tilde{\alpha}}$-point dense connectivity $L_{\tilde{\alpha}}$ without vacancies, represented by an array $\vec{i}_{\tilde{\alpha}}$. Then, connectivity $\alpha$ precedes connectivity $\beta$ if $L_{\tilde{\alpha}}$ exceeds the corresponding number $L_{\tilde{\beta}}$ of connectivity $\beta$. This provides only a partial ordering; it remains to order the set of connectivities with $L_{\tilde{\alpha}}=L_{\tilde{\beta}}$. This remaining ordering will depend on the positions of the zeros in $\vec{i}_{\alpha}$, and on the connectivity $\tilde{\alpha}$ of the remaining dense configuration.

(2) Form an $L_{\alpha}$-bit binary number $B_{\alpha}$ with 0 (1) on position $k$ if $i_{\alpha}(k) \neq 0 \quad\left(i_{\alpha}(k)=0\right)$. We can now specify that connectivity $\alpha$ precedes connectivity $\beta$ if $L_{\alpha}=L_{\beta}$ and $B_{\alpha}<B_{\beta}$.

(3) The remaining task is to order the dense connectivities $\tilde{\alpha}$. The first part is to find the position $n_{\tilde{\alpha}}$ of the loop segment connecting to the loop segment on position 1, that is, the number that satisfies $i_{\tilde{\alpha}}(1)=i_{\tilde{\alpha}}\left(n_{\tilde{\alpha}}\right)$. Then we specify that dense connectivity $\tilde{\alpha}$ precedes $\tilde{\beta}$ if $n_{\tilde{\alpha}}<n_{\tilde{\beta}}$.

(4) If $n_{\tilde{\alpha}}=n_{\tilde{\beta}}$, we define an $L_{\tilde{\alpha}^{\prime}}=L_{\tilde{\alpha}}-2$-point dense connectivity $\tilde{\alpha}^{\prime}$ by removing positions 1 and $n_{\tilde{\alpha}}$ from $\tilde{\alpha}$. The remaining ordering is provided by the recursive application of the last two steps, adding primes at each new iteration, until a decision is found.

The enumeration on the basis of this ordering requires only some trivial bookkeeping, involving numbers of connectivities of the relevant types, using methods presented in Refs. [21] and [36]. An inverse algorithm that derives an array $\vec{i}_{\alpha}$ for a given connectivity number or transfer matrix index was constructed similarly.

\section{CROSSOVER AND THE DENSE PHASE}

We investigate the influence of various perturbations with respect to the branch 2 models on the honeycomb and the square lattice.

\section{A. Attractions between loop segments on the square lattice}

We choose the plus signs in Eq. (9), while noting that the sign of $u$ is irrelevant because the number of $u$-type vertices is even in the systems of interest. Since the number of $v$-type vertices in a loop wrapping a cylinder with odd $L$ is also odd, we have to keep in mind that the sign of $v$ matters for odd system sizes.

Denoting the vertex weights at the branch 2 and the branch 4 points $\left(u^{(2)}, v^{(2)}, w^{(2)}\right)$ and $\left(u^{(4)}, v^{(4)}, w^{(4)}\right)$ respectively, we interpolate between the branch 2 and the branch 4 points and, also, extrapolate, by varying $p$ in

$$
\begin{gathered}
u(p)=(1-p) u^{(2)}+p u^{(4)}, \\
v(p)=(1-p) v^{(2)}+p v^{(4)}, \\
w(p)=(1-p) w^{(2)}+p w^{(4)} .
\end{gathered}
$$

We varied $p$ in the range $-0.5 \leqslant p \leqslant 1.5$ and calculated the scaled magnetic gap $X_{h}(p, L)$. Plots of this quantity as a function of $p$, for several values of the finite-size parameter $L$, are shown in Fig. 3. The main effect of increasing $p$ is that the weight $w$, which controls the attraction between neighboring loops, also increases.

As a result, the loop configuration becomes denser, and at $p=1$ it condenses into a state with Ising order as mentioned in Sec. II B. One observes that, for most $n$, there are clearly two different intersections of the curves for different $L$, near $p=0$ and $p=1$. For $p=0$ the steepest curves are those with the smallest $L$ of the curves for different $L$. For $p=1$ this situation is just the reverse. This shows that the perturbation with respect to branch 2 is irrelevant, in agreement with the expected behavior for the leading thermal exponent according to Eq. (16). In contrast, the perturbation due to the variation of $p$ with respect to the branch 4 point is seen to be relevant. Numerical differentiation to $p$ of the scaled gaps at the branch 4 points for several $n$, and subsequent analysis according to Eq. (21) (with $p$ instead of $P$ ), yielded estimates of $X_{p}$ that are listed in Table I. One observes that the results for $X_{p}$ are close to the known Ising temperature dimension $X_{t}=1$. This confirms the Ising nature of the transition driven by $p$, in line with the conclusion [21] that it takes place independent of the critical background of the dense $\mathrm{O}(n)$ model.

\section{B. The nature of the Ising-ordered phase}

Figure 3 and numerical results for larger values of $p>1$ indicate that the scaled gaps increase approximately linearly with the finite size and, thus, that the magnetic correlation length becomes constant. This corresponds with a magnetic 

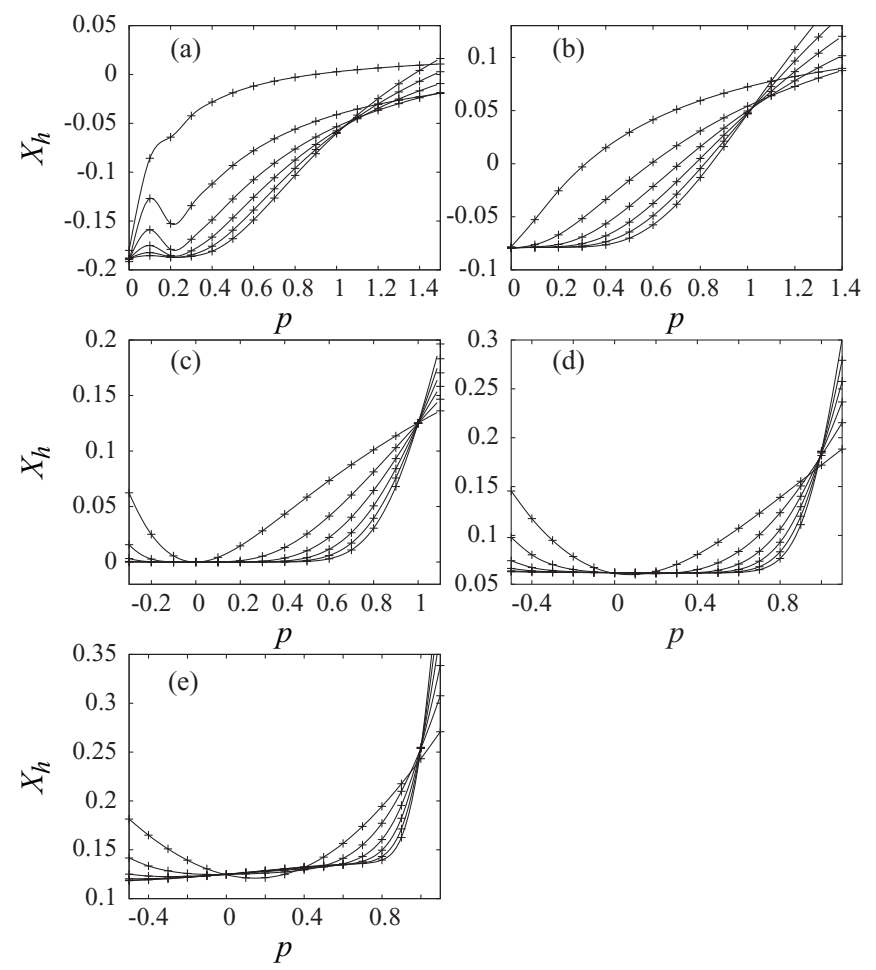

FIG. 3. Scaled magnetic gap $X_{h}$ versus the parameter $p$ that interpolates between branch $2(p=0)$ and branch $4(p=1)$ in the $\mathrm{O}(n)$ model. (a) $n=0$, (b) $n=0.5$, (c) $n=1$, (d) $n=1.5$, and (e) $n=2$. Results are shown for finite sizes $L=2,4, \ldots, 12$. The slope of the curves increases with $L$ on the right-hand side.

correlation function that decays exponentially in the infinite plane. In this respect, the dense phase and the Ising-ordered phase, separated by the Ising line as shown in Fig. 2, are different. Still, these two phases are assumed [21] to share the basic universal properties of the dense $\mathrm{O}(n)$ loop model. We test this assumption by eliminating the reason that the scaled gaps increase sharply at $p=1$ and beyond. The reason is that, near the line of Ising transitions, the loop configurations become so dense that most vertices are of the $w$ type. Since the even sector of the transfer matrix allows only loop configurations that cover an even number of edges in the transfer direction, only configurations of the even sector fit well on a lattice with even $L$. This explains the increase in the gap between the even and the odd sector as the Ising line is approached. Similarly, such dense configurations in the odd sector will fit well only on lattices with odd $L$.

TABLE I. Numerical estimates of the scaling dimensions $X_{p}$ and $X_{w_{2}}$, which belong to the most relevant scaling fields to which $p$ and $w_{2}$ contribute, respectively.

\begin{tabular}{lll}
\hline \hline$n$ & \multicolumn{1}{c}{$X_{p}$} & \multicolumn{1}{c}{$X_{w_{2}}$} \\
\hline 0 & $1.000(1)$ & $1.0(1)$ \\
0.5 & $1.0000(1)$ & $0.99(1)$ \\
1 & $1.000000(2)$ & $0.9999(1)$ \\
1.5 & $1.00001(1)$ & $1.001(1)$ \\
2 & $1.01(1)$ & $0.999(1)$ \\
\hline \hline
\end{tabular}
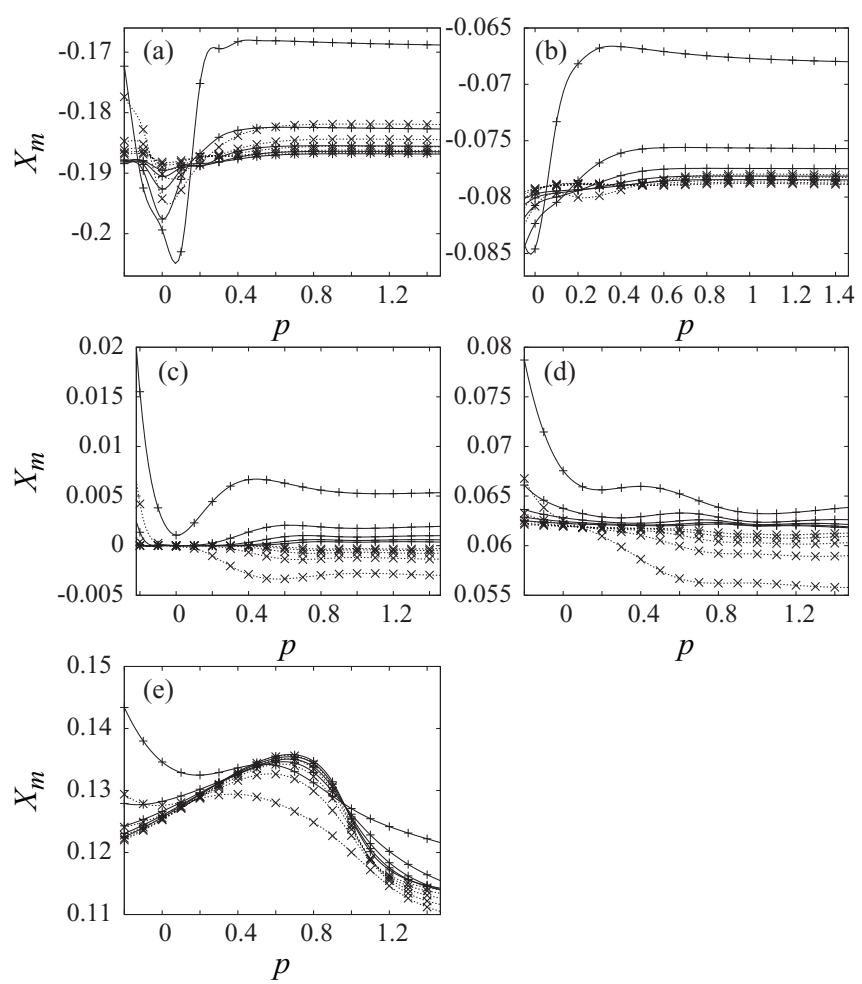

FIG. 4. Scaled magnetic gap $X_{m}(p, L)$ versus the parameter $p$ that interpolates between branch $2(p=0)$ and branch $4(p=1)$ for the cases (a) $n=0$, (b) $n=0.5$, (c) $n=1$, (d) $n=1.5$, and (e) $n=2$ in the $\mathrm{O}(n)$ model. Results are shown for $L=3,4, \ldots, 12$. Dashed curves with X's represent even $L$; solid curves with plus signs, odd $L$. Both sets of curves tend to converge to a common $p$-independent limit when $L$ increases.

To define a type of magnetic gap that excludes these effects of even-odd alternation, one has to select even or odd systems in accordance with the sector. We thus define a magnetic scaled gap $X_{m}(p, L)$ as

$$
X_{m}(p, L) \equiv \frac{\zeta L}{2 \pi} \ln \frac{\sqrt{\Lambda_{0}(L+1) \Lambda_{0}(L-1)}}{\Lambda_{1}(L)}
$$

for odd $L$ and

$$
X_{m}(p, L) \equiv \frac{\zeta L}{2 \pi} \ln \frac{\Lambda_{0}(L)}{\sqrt{\Lambda_{1}(L+1) \Lambda_{1}(L-1)}}
$$

for even $L$.

The results for these scaled magnetic gaps are shown in Fig. 4 as a function of $p$, for several values of $n$. These results indicate that, for $n<2$, the universal character of the magnetic correlations in the dense phase is independent of $p$. In particular, it remains unchanged under the Ising transition and the onset of the Ising-type long-range order.

Next we estimated the associated magnetic scaling dimension $X_{m}$ at the Ising point (branch 4) by fitting the numerical results using Eq. (20) for several $n$. The results are listed in Table II. Here our choice of the sign of $v$ in Eq. (9) follows the change of sign of $w$ such that $v w>0$. The change in sign of $w$ is caused by the change in sign of the common denominator in Eq. (9), which arises because the weight of the empty vertex is normalized to +1 . A consequence of this 
TABLE II. Numerical results for the magnetic scaling dimension $X_{m}$ of branch 4, compared to the exact results for the magnetic dimension $X_{h}$ for branches 2 and 4 . The branch 4 magnetic dimension is equal to the branch 2 dimension plus $1 / 8$.

\begin{tabular}{llll}
\hline \hline$n$ & $X_{h}$ (branch 2) & \multicolumn{1}{c}{$X_{h}$ (branch 4) } & \multicolumn{1}{c}{$X_{m}$} \\
\hline 0 & -0.1875 & -0.0625 & $-0.18749(1)$ \\
0.075 & -0.16865534 & -0.04365534312 & $-0.168653(2)$ \\
0.0875 & -0.16561812 & -0.04061812640 & $-0.16562(2)$ \\
0.1 & -0.16260929 & -0.03760929546 & $-0.162610(2)$ \\
0.125 & -0.15667507 & -0.03167507924 & $-0.156675(2)$ \\
0.5 & -0.07909087 & 0.04590912236 & $-0.07909(1)$ \\
1 & 0 & 0.125 & $0.0000000(1)$ \\
1.5 & 0.061874313 & 0.18687431332 & $0.0618743(1)$ \\
2 & 0.125 & 0.25 & $0.125000(1)$ \\
\hline \hline
\end{tabular}

change of sign is that there will be a jump with value $1 / 8$ in $X_{m}$ near $n=0.087378025$, where the change in sign of $w$ occurs, if the weight $v$ is kept positive. The jump is equal to the "interface dimension" denoted $X_{\text {int, } 1}$ in Ref. [21].

\section{Double bonds in the honeycomb model}

The loop model of Eq. (4) is extended by allowing the edges with one of the three possible orientations, say the vertical edges, to be covered by up to two loop segments. The honeycomb lattice can be decomposed in building blocks consisting of a vertical edge and the pair of vertices at its ends. These units are shown in Fig. 5, together with their weights, which include a factor $z$ per loop segment.

As indicated in Fig. 5, each such unit of the honeycomb lattice can be replaced by a square-lattice vertex. This substitution maps the honeycomb model with double bonds on an $\mathrm{O}(n)$ loop model on the square lattice, but the vertex weights, also shown in Fig. 5, are not of the form of Eq. (6) because the weights of the $u$ - and $w$-type vertices depend on their orientation. Expressed in the enlarged set of square-lattice weights, the partition sum takes the form

$$
Z_{\text {loop }}=\sum_{\mathcal{G}} u_{1}^{N_{u_{1}}} u_{2}^{N_{u_{2}}} v^{N_{v}} w_{1}^{N_{w_{1}}} w_{2}^{N_{w_{2}}} n^{N_{l}},
$$

where the indices to $u$ and $w$ indicate the orientation of the vertex. The vertex weight $z^{\prime}=w_{2}$ describes a double bond covering a lattice edge. The resulting connectivities are,

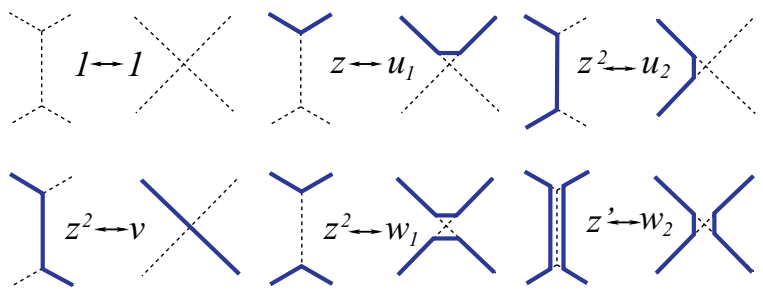

FIG. 5. (Color online) Vertex weights of the $\mathrm{O}(n)$ loop model on the square lattice as obtained by a mapping on a honeycomb lattice. These vertices allow the occupation of vertical edges of the honeycomb lattice by two loop segments. The resulting vertex weights allow for a factor $z$ per loop segment on the honeycomb lattice. however, still of the nonintersecting loop type, which means that the two loop segments on an edge do not cross or mutually connect.

We evaluated the scaled gaps according to Eqs. (18) and (19) of the model of Eq. (25) for several values of the finite size $L$, using the weights $u_{1}, u_{2}, v$, and $w_{1}$ as obtained from the equivalence with the branch 2 point of the honeycomb model with $z$ according to Eq. (5). The influence of double bonds was determined by including several nonzero values of the weight $z^{\prime}=w_{2}$. The calculation of the scaled gaps used the geometric factor $\zeta=2 / \sqrt{3}$ for the honeycomb lattice. Since the additional weight $z^{\prime}$ introduces anisotropy, the asymptotic conformal symmetry is broken, and the scaled gaps for $z^{\prime} \neq 0$ no longer directly relate to the scaling dimension $X_{h}$. For this reason we add a tilde and denote the scaled gaps as $\tilde{X}_{h}\left(z^{\prime}, L\right)$. The results are shown in Fig. 6.

While the interpretation of the scaled gap in terms of the scaling dimension $X_{h}$ is no longer valid, the intersections on the right-hand side of these figures, with slopes increasing with $L$, still indicate that a phase transition takes place, resembling the Ising-like ordering for the square lattice model in Sec. IV A. The Ising character of this transition was verified by means of numerical differentiation of $\tilde{X}_{h}\left(w_{2}, L\right)$ with respect to $w_{2}$ in the intersection points and finite-size scaling, analogous to the analysis of $X_{p}$ in Table I. The numerical estimates of the scaling dimension $X_{w_{2}}$ for several $n$ are included in Table I. They are close to the Ising temperature dimension $X_{t}=1$.
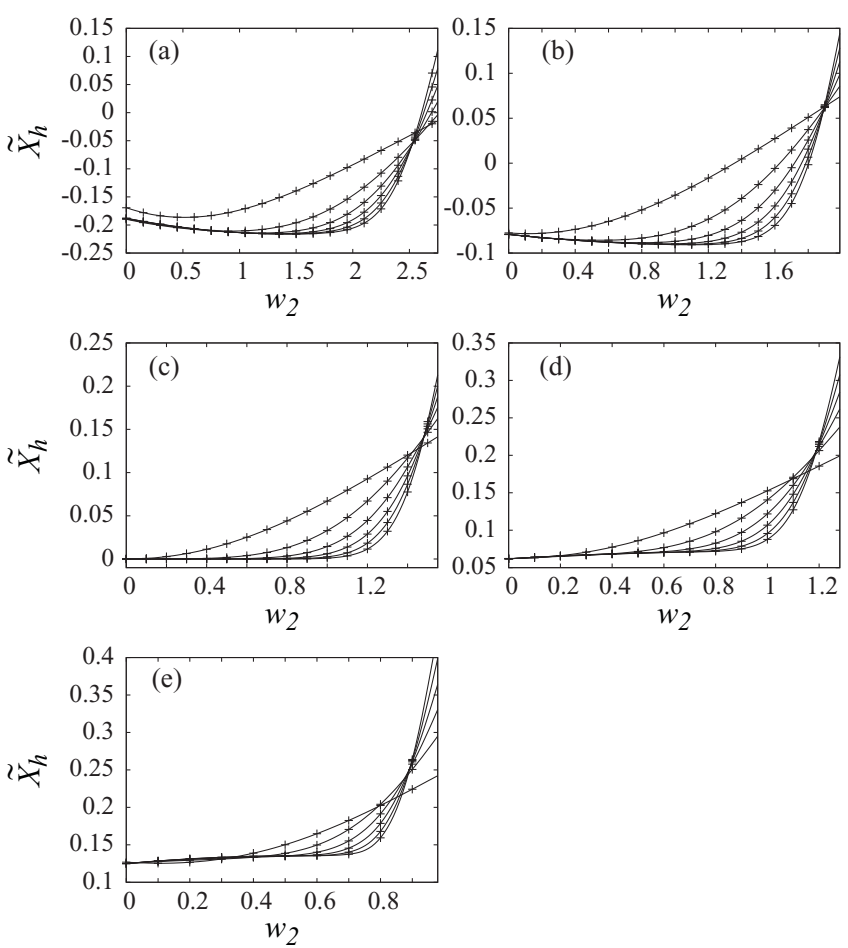

FIG. 6. Scaled magnetic gap $\tilde{X}_{h}\left(w_{2}, L\right)$ versus the weight $w_{2}$ of a double bond covering an edge of the honeycomb lattice for the $\mathrm{O}$ (n) model with (a) $n=0$, (b) $n=0.5$, (c) $n=1$, (d) $n=1.5$, and (e) $n=2$. Results are shown for finite sizes $L=2,4, \ldots, 12$. The slope of the curves increases with $L$ on the right-hand side. 


\section{Cubic anisotropy}

The introduction of cubic vertices into the $\mathrm{O}(n)$ model of Eq. (6) modifies the partition function as follows:

$$
Z_{\text {loop }}=\sum_{\mathcal{G}} u^{N_{u}} v^{N_{v}} w^{N_{w}} c^{N_{c}} n^{N_{l}},
$$

where $N_{c}$ is the number of vertices of type $c$. For $w=0, v=u$, and $c=u^{2}$ it reduces, apart from a multiplicative constant, to the partition sum of the cubic model, Eq. (11). Thus, Eq. (26) can interpolate between the nonintersecting loop model and the cubic model.

We investigate the effect of cubic perturbations by varying $c$, while keeping the other vertex weights fixed at their branch 2 values. The results for the scaled gaps are shown in Fig. 7. For $n=0$, the vanishing loop weight prevents the introduction of cubic vertices, so that the results do not depend on $c$. The slopes of the curves with $0<n<2$ are seen to increase with $L$ near $c=0$, which shows that the cubic perturbation is relevant. Furthermore, some of the plots display two more sets of intersections, of which the middle ones are indicative of a stable fixed point, and the rightmost ones of an unstable fixed point, resembling that of the Ising-like transition induced by the $w$-type vertices. These results are consistent with the interpretation that, in the range attracted by the stable fixed point, the scaled gap converges to $X_{h}=0$ for $0<n<2$ in the range $c>0$. This was confirmed by numerical extrapolations at some values of $c$, all of which satisfied $X_{h} \leqslant 0.01$.

To numerically determine the exponent responsible for the cubic crossover, we performed a numerical differentiation of the scaled magnetic gap $X_{h}(c, L)$ with respect to the weight $c$ of the cubic vertex at point $c=0$ for several values of $n$. The finite-size data for this derivative were subsequently analyzed according to Eq. (21), with $P$ replaced by $c$. The resulting estimates of $X_{c}$ are listed in Table III, together with the Coulomb gas predictions.

\section{E. Crossing bonds}

We next introduce, starting from the branch 2 lowtemperature points of the square-lattice loop model, a nonzero weight of the crossing-bond vertex. We calculated the scaled gaps for a range of values of the weight $x$ and for several

TABLE III. Scaling dimensions $X_{c}$, associated with a cubic perturbation, and $X_{x}$, associated with crossing bonds for the case of a dense $\mathrm{O}(n)$ phase. These results are obtained by numerical differentiation of the scaled magnetic gap $X_{h}(L)$ at the branch 2 point. The vanishing of some numerical results for $n=0$ and 1 is an artifact due to suppression of the respective critical amplitudes.

\begin{tabular}{llllll}
\hline & \multicolumn{2}{c}{ Coulomb gas (branch 2) } & & \multicolumn{2}{c}{ Numerical results } \\
\cline { 2 - 3 } \cline { 5 - 6 }$n$ & \multicolumn{1}{c}{$g$} & $X_{x}=X_{c}$ & & \multicolumn{1}{c}{$X_{c}$} & \multicolumn{1}{c}{$X_{x}$} \\
\hline 0 & 0.5 & 0.75 & & 0 & $0.75001(1)$ \\
0.5 & 0.58043 & 1.00922 & & $1.009(1)$ & $1.0092(2)$ \\
1 & $2 / 3$ & 1.25 & & 0 & 0 \\
1.5 & 0.76995 & 1.50552 & $1.505(1)$ & $1.5055(3)$ \\
2 & 1 & 2 & $2.000(2)$ & $1.998(2)$ \\
\hline \hline
\end{tabular}
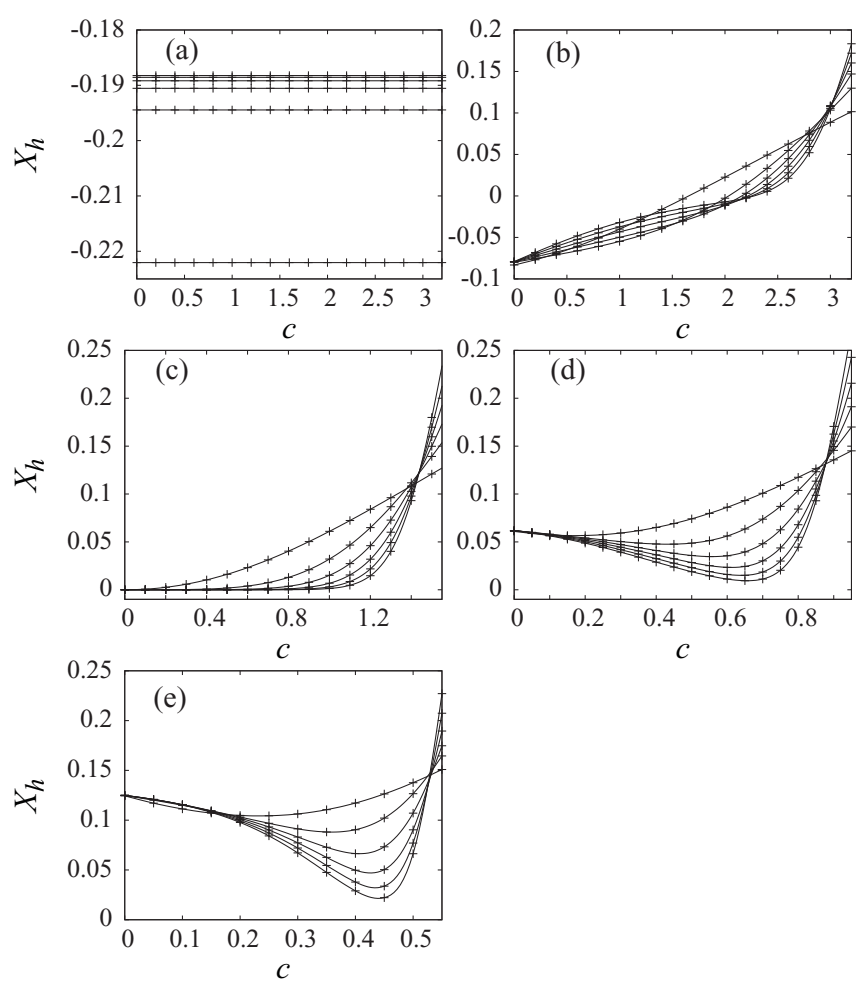

FIG. 7. Scaled magnetic gap $X_{h}$ versus the weight $c$ of cubic vertices introduced into the dense phase of the $\mathrm{O}(n)$ nonintersecting loop model according to Eq. (26). Results are shown for finite sizes $L=2,4, \ldots, 12$. (a) $n=0$. The weight $c$ has no effect because the loop weight $n=0$ does not allow a nonzero density of $c$-type vertices. The gap increases as a function of $L$. (b) $n=0.5$; (c) $n=1$; (d) $n=1.5$; (e) $n=2$. The slope of the curves increases with $L$ on the right-hand side.

values of $n$. The results are shown in Fig. 8. These data indicate that, for $n<2$ and a range of $x>0$, crossover occurs to a different universality class of dense intersecting loop models, with a magnetic exponent that is different from that of nonintersecting loop models for $n \neq 1$. This interpretation is in line with a prediction of Jacobsen et al. [30] in terms of exact results $[31,32]$. We have attempted to find the conformal anomaly $c_{a}$ and the magnetic scaling dimension $X_{h}$ from the finite-size data for a few points in the phase diagram. Estimates for $c_{a}$ are obtained by fitting $f(L)=f(\infty)+\pi c_{a} /\left(6 L^{2}\right)$ to transfer-matrix results for the free energy using three subsequent values of $L$. These estimates display slow apparent convergence and are thus hard to extrapolate. Extrapolation was done assuming finite-size dependence as $L^{-2}$. The results are listed in Table IV. As a tentative error margin we quote ten times the difference between the last two extrapolations. Also, the data for $X_{h}$ were hard to extrapolate; we simply quote the result $X_{h}(L)$ obtained from Eq. (19) for $L=14$, with a tentative error margin of $10 \times\left[X_{h}(14)-X_{h}(12)\right]$.

We also performed numerical differentiations of the scaled magnetic gaps $X_{h}(x, L)$ with respect to the weight $x$ of the crossing-bond vertex at the point $x=0$ for several $n$. The finite-size data were subsequently analyzed according to Eq. (21), with $P$ replaced by $x$. This yielded estimates of $X_{x}$ that are listed in Table III, together with the Coulomb gas predictions. 
TABLE IV. Numerical estimates of the conformal anomaly $c_{a}$ and the magnetic scaling dimension $X_{h}$ in the dense $\mathrm{O}(n)$ phase with crossing bonds.

\begin{tabular}{lccc}
\hline \hline$n$ & $x$ & $c_{a}$ & $X_{h}$ \\
\hline 0 & 0.5 & $-1.42(26)$ & $-0.066(29)$ \\
0 & 0.8 & $-1.36(29)$ & $-0.053(15)$ \\
0.5 & 0.4 & $-0.64(19)$ & $-0.036(13)$ \\
0.5 & 0.6 & $-0.65(10)$ & $-0.031(9)$ \\
1 & 0.8 & $-0.01(10)$ & $0.000(2)$ \\
1 & 1.1 & $0.05(64)$ & $0.011(50)$ \\
1.5 & 0.6 & $0.55(2)$ & $0.031(3)$ \\
1.5 & 0.8 & $0.53(11)$ & $0.025(3)$ \\
1.5 & 1.0 & $0.52(35)$ & $0.025(30)$ \\
2 & 0.3 & $1.000(2)$ & $0.095(1)$ \\
2 & 0.6 & $0.998(46)$ & $0.067(1)$ \\
2 & 0.9 & $0.97(29)$ & $0.046(35)$ \\
\hline \hline
\end{tabular}

\section{DISCUSSION}

The numerical results presented in Sec. IV A for the effect of the $w$-type vertex, representing loop-loop attractions, on the low-temperature phase of the square-lattice model agree with the predicted [21] behavior. As indicated by the curves in Fig. 3, these attractions are irrelevant in the whole range interpolating between the branch 4 and the branch 2 points. In contrast, they are relevant in the equivalent tricritical $\mathrm{O}(n)$ model with vacancies [18]. The latter model is obtained by
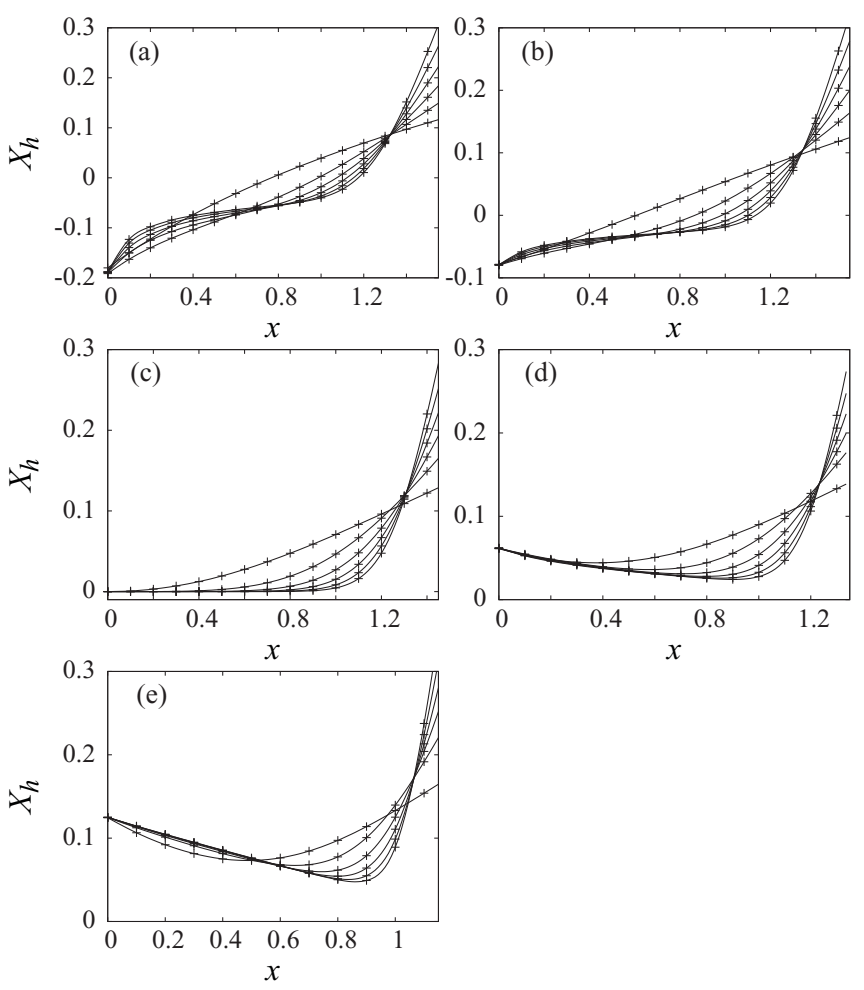

FIG. 8. Scaled magnetic gap $X_{h}$ versus the weight of the crossingbond vertex, for the cases (a) $n=0$, (b) $n=0.5$, (c) $n=1$, (d) $n=$ 1.5 , and (e) $n=2$. Results are shown for finite sizes $L=2,4, \ldots, 12$. The slope of the curves increases with $L$ on the right-hand side. summing out part of the loops, which thus yields a less dense system that is far more susceptible to attractions between the loops. The irrelevance of the double bonds in the critical $\mathrm{O}(n)$ model, as illustrated by the figures in Sec. IV C, is in agreement with the occurrence of multiple bonds on the lattice edges in the high-temperature expansions of $\mathrm{O}(n)$ models with spin-spin interactions of a different form than Eq. (2), that is, the model with interaction energies $J \vec{s}_{i} \cdot \vec{s}_{j}$ [1]. It is also in line with the existence of special cases of exactly solvable $\left(N_{\alpha}, N_{\beta}\right)$ models [37] for $N_{\alpha}, N_{\beta}=q$ in the $q$-state Potts universality class and the relation [38] between Potts and loop models, which then generates double bonds.

Furthermore, the numerical results confirm that the looploop attractions become relevant at branch 4 and that the transition that takes place at this point is Ising-like. Apart from the explanation of this transition in terms of the onset of long-range order of the dual Ising spins, it may be worthwhile to mention that the type of phase diagram in Fig. 2, including the Ising line, is reproduced by an Ising model with vacancies quoted in Ref. [18]. In the latter case, the Ising line corresponds to the onset of phase separation between a phase dominated by Ising spins and a phase dominated by vacancies.

The analysis of the $\mathrm{O}(n)$ magnetic dimension $X_{m}$ presented in Sec. IV B confirms that the low-temperature $\mathrm{O}(n)$ universal character remains unaffected across the Ising transition on branch 4 . This result was obtained by means of a careful formulation of the correlation function associated with $X_{m}$, which takes into account the even- or oddness of the system, even in the limit of infinite size.

On the basis of results [30-32] for loop models that allow crossing bonds, doubts have arisen regarding to what extent the behavior found for the exactly solvable models of branch 2 of the honeycomb [6], the square [11], and the triangular lattice [39] is representative for the low-temperature $\mathrm{O}(n)$ phase. Since multiple bonds arise in a natural way in graph expansions of more general spin $\mathrm{O}(n)$ models, we studied the effect of double bonds in Sec. IV C. This perturbation was found to be irrelevant for branch 2 of the model on the honeycomb lattice. Its effect appears to be very similar to that of loop-loop attractions due to the type $w$ vertex in the case of the square lattice. The similarity includes the Ising-like transition that takes place at a sufficiently large weight of the double bonds.

The Coulomb gas prediction that the cubic perturbation is relevant for $n<2$ is quantitatively confirmed by the results for $X_{c}$ in Table III only for $n=0.5$ and 1.5 . We attribute the vanishing numerical results for $n=0$ and 1 to vanishing amplitudes associated with the cubic perturbation. The numerical result $X_{c}=0$ at $n=0$ is due to the fact that the zero loop weight excludes type $c$ vertices even at nonzero fugacity. For $n=1$, the loop weights are equal to 1 , so that the distinction between type $z$ and type $c$ vertices disappears, and so do the amplitudes associated with the cubic perturbation. Taking into account this explanation of the deviating results for $n=0$ and 1 , we conclude that there is satisfactory agreement with the Coulomb gas predictions.

As mentioned in Sec. II C, the relevance of cubic perturbations in the low-temperature phase of the square-lattice model of Eq. (11) for $n<2$, which was confirmed in Sec. IV D, 
seems very peculiar in relation to the absence of a crossover to cubic behavior for the honeycomb model. In view of the mutually incompatible values of $X_{h}$, the conclusion that the dense phases of the partition sum of Eq. (11) display different universal behavior for the square and honeycomb lattices is inescapable and has to be attributed to the low coordination number of the honeycomb lattice.

One may wonder if a similar paradox occurs in the spin representation of the model, for which our physical intuition may provide further insight. The equivalence with Eq. (11) applies only to cases where $n$ is a positive integer. The case $n=1$ fails to provide more clarity because the amplitude associated with $c$ vanishes. For $n=2$ we do expect a phase transition to a long-range-ordered state in the spin model described by Eq. (10) at sufficiently low temperatures, but the condition $e^{M} \cosh K=1$ excludes this low-temperature range from the loop representation of Eq. (11). There is no apparent conflict with the expected behavior of the cubic spin model.

The introduction of the cubic vertices into the loop model on the square lattice in Sec. IV D yielded results that are consistent with the interpretation that the scaled gaps converge to 0 for $n<2$ in a range $c>0$. This interpretation is in line with the expected long-range order of spin models with a cubic perturbation at low temperatures.

Furthermore, we note that the mapping of the honeycomb model on the square lattice model presented in Sec. IV C can be extended to include cubic vertices on the square lattices, which corresponds to mutually connecting double bonds on the vertical edges of the honeycomb model. Thus, cubic crossover will occur on a suitably generalized honeycomb model.

Also in the case of perturbations introduced by the crossingbond vertex we find a satisfactory agreement with the Coulomb gas predictions. And in this case the amplitude $a$ in Eq. (20) due to the perturbation vanishes at $n=1$ (see Table III), which explains the vanishing of the corresponding numerical result. For $n=1.5$ the amplitude is still rather low [see Fig. 8(d)], but the numerical differentiation method is sufficiently sensitive to determine the scaling dimension $X_{x}$.

The scaled gaps in the dense phase perturbed by crossing bonds appear difficult to analyze. While our range of finite sizes is insufficient for reliable extrapolations, the data seem to be in line with $X_{h}=0$ and the occurrence of logarithmic factors as predicted for crossing-bond models [30-32]. Moreover, the results for the conformal anomaly listed in Table IV show a trend consistent with $c_{a}=n-1$ as predicted by Martins et al. [31].

In conclusion, our results confirm the phase diagram of the $\mathrm{O}(n)$ model as conjectured in Ref. [21], in particular, the Ising transition between the dense and the Ising-ordered phases and its Ising scaling dimension. Cubic anisotropy and crossing bonds are proved numerically to be relevant and introduce crossover to different universal behavior in the lowtemperature (dense) phase. While our numerical analyses were restricted to one perturbation at a time, in a possible extension one might also consider the simultaneous effects of cubic vertices and crossing bonds. Although the flow away from branch 2 is initially still determined by the single Coulomb gas exponent $X_{c}=X_{x}$, at sufficiently strong perturbations one may expect a behavior dependent on the relative strength of the two perturbations, including a phase boundary between the long-range ordered cubic ground state and the algebraic phase of the crossing-bond model. In relation to this question, it is of interest that further information may possibly be obtained from exactly solvable cases [14,17] in which both weight $x$ and weight $c$ in Fig. 1 are nonzero. In this context we note that there are also other exactly solvable cases $[14,16,17]$ that intersect with our parameter space for $x=0, c \neq 0$ as investigated in Sec. IV D.

Finally, we remark that our numerical results display so-called nonuniversal behavior as a function of the various perturbations in the $n=2$ models, as expected from the mapping between the cubic model and, for example, the Ashkin-Teller model [28] and the equivalence of the latter model to the eight-vertex model [40-42].

\section{ACKNOWLEDGMENTS}

We are indebted to Professors B. Nienhuis and J. H. H. Perk for valuable discussions. W.G. acknowledges the hospitality extended to him by the Lorentz Institute. This work is supported by the Lorentz Fund, the NSFC under Grant No. 10675021, the NCET, and the HSCC (High Performance Scientific Computing Center) of the Beijing Normal University.
[1] H. E. Stanley, Phys. Rev. Lett. 20, 589 (1968).

[2] E. Domany, D. Mukamel, B. Nienhuis, and A. Schwimmer, Nucl. Phys. B 190, 279 (1981).

[3] P. G. de Gennes, Scaling Concepts in Polymer Physics (Cornell University, Ithaca, NY, 1979).

[4] B. Nienhuis, in Fundamental Problems in Statistical Mechanics VII, edited by H. van Beijeren (Elsevier, Amsterdam, 1990), p. 255.

[5] B. Duplantier and H. Saleur, Phys. Rev. Lett. 59, 539 (1987).

[6] B. Nienhuis, Phys. Rev. Lett. 49, 1062 (1982); J. Stat. Phys. 34, 731 (1984).

[7] R. J. Baxter, J. Phys. A 19, 2821 (1986); 20, 5241 (1987).
[8] M. T. Batchelor and H. W. J. Blöte, Phys. Rev. Lett. 61, 138 (1988); Phys. Rev. B 39, 2391 (1989).

[9] J. Suzuki, J. Phys. Soc. Jpn. 57, 2966 (1988).

[10] H. Kunz and F. Y. Wu, J. Phys. A 21, L1141 (1988); see Ref. [22] for an erratum.

[11] M. T. Batchelor, B. Nienhuis, and S. O. Warnaar, Phys. Rev. Lett. 62, 2425 (1989).

[12] S. O. Warnaar, M. T. Batchelor, and B. Nienhuis, J. Phys. A 25, 3077 (1992).

[13] S. O. Warnaar, P. A. Pearce, K. A. Seaton, and B. Nienhuis, J. Stat. Phys. 74, 469 (1994).

[14] C. L. Schultz, Phys. Rev. Lett. 46, 629 (1981).

[15] V. A. Fateev, Sov. J. Nucl. Phys. 33, 761 (1981). 
[16] J. H. H. Perk and F. Y. Wu, Physica A (Amsterdam) 138, 100 (1986).

[17] J. H. H. Perk and C. L. Schultz, in Proceedings, RIMS Symposium on Non-Linear Integrable Systems, edited by M. Jimbo and T. Miwa (World Scientific, Singapore, 1983), p. 135; and in Yang-Baxter Equation in Integrable Systems, edited by M. Jimbo (World Scientific, Singapore, 1990), p. 326.

[18] B. Nienhuis, W.-A. Guo, and H. W. J. Blöte, Phys. Rev. E 78, 061104 (2008).

[19] L. P. Kadanoff, J. Phys. A 11, 1399 (1978).

[20] B. Nienhuis, in Phase Transitions and Critical Phenomena, vol. 11, edited by C. Domb and J. L. Lebowitz (Academic Press, London, 1987).

[21] H. W. J. Blöte and B. Nienhuis, J. Phys. A 22, 1415 (1989); B. Nienhuis, Int. J. Mod. Phys. B 4, 929 (1990).

[22] W.-A. Guo, H. W. J. Blöte, and F. Y. Wu, Phys. Rev. Lett. 85, 3874 (2000).

[23] R. J. Baxter, J. Phys. A 19, 2821 (1986); 20, 5241 (1987).

[24] W.-A. Guo, H. W. J. Blöte, and B. Nienhuis, Int. J. Mod. Phys. C 10, 301 (1999).

[25] H. W. J. Blöte, M. T. Batchelor, and B. Nienhuis, Physica A (Amsterdam) 251, 95 (1998).

[26] W.-A. Guo, B. Nienhuis, and H. W. J. Blöte, Phys. Rev. Lett. 96, 045704 (2006).

[27] W.-A. Guo, H. W. J. Blöte, and Y.-Y. Liu, Commun. Theor. Phys. 41, 911 (2004).
[28] H. W. J. Blöte and M. P. Nightingale, Physica A (Amsterdam) 129, 1 (1984).

[29] W.-A. Guo, X.-F. Qian, H. W. J. Blöte, and F. Y. Wu, Phys. Rev. E 73, 026104 (2006).

[30] J. L. Jacobsen, N. Read, and H. Saleur, Phys. Rev. Lett. 90, 090601 (2003).

[31] M. J. Martins, B. Nienhuis, and R. Rietman, Phys. Rev. Lett. 81, 504 (1998); M. J. Martins and B. Nienhuis, J. Phys. A 31, L723 (1998).

[32] J. de Gier and B. Nienhuis, J. Stat. Mech.: Theor. Exp. (2005) P01006.

[33] J. L. Cardy, J. Phys. A 17, L385 (1984).

[34] For a review, see, e.g., M. P. Nightingale in Finite-Size Scaling and Numerical Simulation of Statistical Systems, edited by V. Privman (World Scientific, Singapore, 1990).

[35] H. W. J. Blöte and B. Nienhuis, Physica A (Amsterdam) 160, 121 (1989).

[36] H. W. J. Blöte and M. P. Nightingale, Physica A (Amsterdam) 112, 405 (1982).

[37] H. Au-Yang and J. H. H. Perk, Int. J. Mod. Phys. A 7,1025 (1992).

[38] R. J. Baxter, S. B. Kelland, and F. Y. Wu, J. Phys. A 9, 397 (1976).

[39] Y. M. M. Knops, B. Nienhuis, and H. W. J. Blöte, J. Phys. A 31, 2941 (1998).

[40] C. Fan, Phys. Rev. B 6, 902 (1972).

[41] F. J. Wegner, J. Phys. C 5, L131 (1972).

[42] R. J. Baxter, Phys. Rev. Lett. 26, 832 (1971). 